\title{
Relação de estratificação como indicador do sequestro de carbono em macroagregados de Latossolo sob plantio direto
}

\author{
Stratification ratio as soil carbon sequestration indicator in macroaggregates of Oxisol under no-tillage
}

\author{
Ademir de Oliveira Ferreira' ${ }^{\mathrm{I}}$ João Carlos de Moraes SáII Mônica Gabrielle Harms ${ }^{\mathrm{II}}$ \\ Simone MiaraII Clever Briedis" Caio Quadros Netto II Josiane Burkner dos Santos ${ }^{\text {II }}$ \\ Lutécia Beatriz dos Santos Canalli ${ }^{\mathrm{III}}$ Carlos Tadeu dos Santos Dias ${ }^{\text {IV }}$
}

\section{RESUMO}

A redução no conteúdo de carbono (C) nas camadas mais profundas do solo indica a estratificação entre a camada superficial e as subsuperficiais, devido à adição contínua de $C$ pelos resíduos culturais na superfície. O objetivo deste trabalho foi avaliar a variação $(\Delta)$ da relação de estratificação (RE) de carbono como indicadora do sequestro de $C$ total e particulado em macroagregados de dois Latossolos de classes texturais diferentes, manejados em sistema plantio direto. Os ensaios foram desenvolvidos em delineamento inteiramente casualizado com doze repetições. Os fatores analisados foram: (a) dois solos (Latossolo Vermelho Distrófico típico com classe textural franco-argilo-arenosa e Latossolo Vermelho Distrófico típico com classe textural franco-argilosa); (b) duas camadas de amostragem $(0-5$ e 5-20 cm de profundidade); (c) duas épocas de amostragem ( $E_{1}$-outubro de 2007; E -setembro de 2008). Observou-se relação linear $e$ significativa entre o delta RE com a taxa de sequestro de $C$ total em macroagregados no Latossolo Vermelho com classe textural franco argilo arenosa $\left(R^{2}=0,78^{* *}\right)$ e franco argilosa $\left(R^{2}=0,81^{* *}\right)$, indicando ter havido sequestro de $C e$ confirmando ser este um indicador sensível da taxa de sequestro de $C$ no solo em macroagregados.

Palavras-chave: solos subtropicais, textura do solo, matéria orgânica.

\section{ABSTRACT}

The decrease in soil organic carbon (SOC) content in deeper layers indicates the occurrence of stratification between the surface layer and subsurface layer of soil due to continuous $C$ addition by crop residues. The objective was to evaluate the change (delta) of stratification (RE) of carbon (C) as an indicator of $C$ sequestration and soil total and particulate soil in macroaggregates of two soils (Typic Hapludox) with different textures, managed in no-tillage system. The tests were developed in completely randomized design twelve repetitions.Analyzed factors were: (a) two soils: Oxisol with medium texture and Oxisol with clay texture, (b) sampling depth: $0-5$ and 5-20cm, (c) two sampling times (October 2007- $T_{1}$, September 2008$\left.T_{2}\right)$. The results were submitted to variance analysis by program SISVAR 5.3, using the Tukey test at 5\% significance. There was a significant linear relationship between the $\triangle R E$ and carbon sequestration rate total macroaggregates Oxisol with medium texture $\left(R^{2}=0.78^{* *}\right)$ and Oxisol with clay texture $\left(R^{2}=0.81^{* *}\right)$ indicating that there was $C$ sequestration confirming to be a sensitive indicator of the rate of $C$ sequestration in soil macroaggregates.

Key words: subtropical soils, Oxisol, soil texture, organic matter.

\section{INTRODUÇÃO}

Em geral, nos ecossistemas naturais, o conteúdo de carbono (C) diminui desde a superfície em direção às camadas mais profundas do solo, resultando em sua estratificação no perfil. Este fenômeno, observado em áreas com vegetação natural, ocorre devido ao efeito combinado do continuado aporte superficial dos resíduos vegetais animais e a ausência de mobilização do solo. Fenômeno semelhante

IDepartamento de Solos, Universidade Federal de Santa Maria (UFSM), Av. Roraima 1000, 97105-900, Santa Maria, RS, Brasil. Email: aoferreira1@yahoo.com.br. Autor para correspondência.

IIDepartamento de Solos e Engenharia Agrícola, Universidade Estadual de Ponta Grossa (UEPG), Ponta Grossa, PR, Brasil.

III Instituto Paranaense de Assistência Técnica e Extensão Rural - EMATER, Ponta Grossa, PR, Brasil.

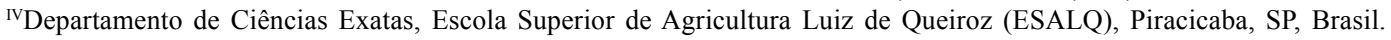


ocorre no sistema plantio direto (SPD) utilizado de forma ininterrupta (SÁ \& LAL, 2009; FERREIRA et al., 2011).

A camada superficial é a interface vital entre o solo e a atmosfera, a qual recebe grande parte dos fertilizantes e agroquímicos aplicados aos cultivos. Ela está exposta ao impacto das gotas de chuvas, concentra a maior atividade biológica e é responsável pelas partições de água, gases e energia no agroecossistema. Por essas razões, a relação de estratificação do carbono pode ser usada como indicador da qualidade do solo, porque a matéria orgânica desempenha múltiplas funções, com destaque para o incremento da sua resistência à erosão, ao aumento da infiltração da água e à retenção de nutrientes (FRANZUEBBERS, 2010).

A relação de estratificação (RE) do $\mathrm{C}$ orgânico total (COT) é expressa como uma razão deste elemento entre camadas do solo, que reflete o grau de organização e o funcionamento dos ecossistemas. $\mathrm{O}$ princípio é comparar uma camada superficial, com elevada influência das práticas de manejo antrópicas, com outra camada com baixo impacto dessas práticas. SÁ \& LAL (2009) constataram estreita relação entre a RE (0-5: 5-10cm) com a taxa de sequestro de C na camada de $0-10 \mathrm{~cm}$, sugerindo que ambos os processos ocorrem concomitantemente e, por isso, a RE poderia ser utilizada como indicador do sequestro de $\mathrm{C}$ em áreas sob SPD.

A RE necessita ser investigada sob clima tropical e subtropical, especialmente quanto ao tipo de solo, à espessura das camadas, ao valor crítico e sua relação com o tempo de adoção de sistemas de manejo do solo. No Brasil, ainda são escassos os trabalhos investigando a RE em classes de macroagregados de diferentes classes texturais de solo. O objetivo deste trabalho foi avaliar a variação $(\triangle)$ da relação de estratificação (RE) de carbono (C) como indicador do sequestro de $\mathrm{C}$ total e particulado do solo em macroagregados de dois Latossolos com classes texturais diferentes, manejado em sistema plantio direto por longo prazo.

\section{MATERIAL E MÉTODOS}

Descrição da área de estudo

Este trabalho foi desenvolvido em dois solos sob SPD de longa duração na Fazenda Escola Capão da Onça - FESCON, município de Ponta Grossa, PR, Brasil, situada a $990 \mathrm{~m}$ de altitude, nas coordenadas geográficas de $25^{\circ} 05^{\prime} 49^{\prime \prime}$ LS e $50^{\circ} 03^{\prime} 11^{\prime \prime}$ LW. O primeiro solo é classificado como Latossolo Vermelho Distrófico típico (EMBRAPA, 2006) com classe textural franco argilo arenosa (232 $\mathrm{g} \mathrm{kg}^{-1}$ de argila) e com declividade média de 5\%, denominado neste trabalho como LVTM. O segundo solo é classificado como Latossolo
Vermelho Distrófico típico (EMBRAPA, 2006) com classe textural franco argilosa $\left(401 \mathrm{~g} \mathrm{~kg}^{-1}\right.$ de argila) e com declividade média de $7 \%$, denominado neste trabalho como LVTA. As camadas de amostragem foram 0-5cm e 5-20 cm em duas épocas de amostragem (após colheita do trigo, em outubro de $2007-\mathrm{E}_{1}$ - e após o manejo mecânico da aveia preta + ervilhaca, em setembro de $2008-E_{2}$ ). Mais detalhes das características dos solos foram descritos por FERREIRA et al. (2011).

O clima da região é classificado como subtropical úmido, mesotérmico. No período do experimento (outubro de 2007 a setembro 2008), a temperatura média máxima foi de $26^{\circ} \mathrm{C}$ e a mínima de $13^{\circ} \mathrm{C}$, e a pluviosidade total foi de $1.558 \mathrm{~mm}$ (IAPAR, 2008).

As lavouras onde foram coletadas as amostras vinham sendo manejadas em SPD por longo prazo (20 anos) com as seguintes rotações de cultura: trigo/soja/aveia preta + ervilhaca/milho (LVTM); trigo/ milho/aveia preta + ervilhaca/soja (LVTA). A quantidade de resíduos culturais das áreas em estudo foi, em média, de $5,16 \mathrm{Mg} \mathrm{ha}^{-1}$.

Análises químicas (PAVAN et al., 1992) e granulométricas (EMBRAPA, 1997) na camada de 0$20 \mathrm{~cm}$ do solo, realizadas antes da instalação do experimento, encontram-se na tabela 1.

Estabilidade de agregados

A separação das classes de agregados foi realizada pelo pré-tratamento das amostras no campo, de acordo com metodologia descrita em BARRETO et al. (2009), na qual se preconiza a classificação de agregados naturais por tamanho, sendo esses separados em grupos estáveis sob uma força destrutiva manual aplicada, ou seja, foi aplicada um força suficiente para romper o agregado no seu ponto de fraqueza. Em laboratório, as classes de agregados foram separadas por peneiramento úmido, de acordo com metodologia descrita por CASTRO FILHO et al. (1998).

Sequência do fracionamento granulométrico para separação do carbono orgânico particulado (Ø entre $53-250 \mu \mathrm{m})$

Cada classe de agregados (diâmetro entre 8-19, 4- 8, 2-4, 1-2, 0,5-1 e 0,25-0,5mm) foi dividida em duas subamostras, sendo uma parte para determinação do $\mathrm{C}$ orgânico total e a outra para o fracionamento físico para retirada do carbono orgânico particulado (COP), que corresponde à fração da Mo compreendida entre $53 \mu \mathrm{m}-250 \mu \mathrm{m}$ de diâmetro. Na fração particulada foi determinado o conteúdo de C. O fracionamento físico da MO foi realizado de acordo com metodologia descrita por FELLER (1994). 
Tabela 1 - Caracterização química e física do Latossolo Vermelho distrófico típico nas duas classes texturais.

\begin{tabular}{|c|c|c|c|c|c|c|c|c|c|c|c|c|}
\hline $\begin{array}{l}\text { Classe } \\
\text { textural }\end{array}$ & Prof. & $\mathrm{pH}$ & $\mathrm{H}+\mathrm{Al}^{+3}$ & $\mathrm{Al}^{+3}$ & $\mathrm{Ca}^{+2}$ & $\mathrm{Mg}^{+2}$ & $\mathrm{~K}^{+}$ & $\mathrm{P}^{*}$ & $\mathrm{C}$ & Areia & Silte & Argila \\
\hline & $(\mathrm{cm})$ & $\left(\mathrm{CaCl}_{2}\right)$ & $\begin{array}{ll}------ \\
\end{array}$ & 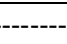 & $\mathrm{nol}_{\mathrm{c}} \mathrm{dm}^{-3}$ & ------ & 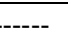 & $\mathrm{mg} \mathrm{dm}^{-3}$ & \multicolumn{4}{|c|}{----------------g kg-1 ---------------- } \\
\hline $\mathrm{LVTM}^{\dagger}$ & $0-20$ & 5,2 & 50 & 2,0 & 31,0 & 20,0 & 3,0 & 1,4 & 14,0 & 667 & 101 & 232 \\
\hline LVTA $^{\dagger \dagger}$ & $0-20$ & 5,0 & 65 & 1,5 & 30,0 & 15,0 & 4,0 & 1,9 & 20,0 & 507,5 & 91,5 & 401 \\
\hline
\end{tabular}

${ }^{\dagger}$ Latossolo Vermelho Distrófico típico, com classe textural franco argilo arenosa ; ${ }^{\dagger \dagger}$ Latossolo Vermelho Distrófico típico, com classe textural franco argilosa $;{ }^{\dagger \dagger}$ Mehlich ${ }^{-1}$.

\section{Determinação de COT e COP}

As amostras de solo foram finamente moídas em gral de porcelana. O conteúdo de COT e COP nas classes de agregados foi determinado por combustão seca, utilizando um analizador elementar de C (TruSpec CN LECO ${ }^{\circledR} 2006$, St. Joseph, EUA). Para determinar a densidade do solo, amostras indeformadas foram coletadas nas camadas de $0-5$ e $5-20 \mathrm{~cm}$, com anéis volumétricos com dimensões de $5 \mathrm{~cm}$ de diâmetro e 4 cm de altura (EMBRAPA, 1997).

Cálculo da relação de estratificação do carbono

A RE foi calculada conforme proposto por Franzluebbers (2002), sendo o conteúdo de C da camada superficial do solo $(0-5 \mathrm{~cm})$ dividido pelo valor daqueles da camada subsuperficial $(5-20 \mathrm{~cm})$. A variação temporal da RE $(\Delta \mathrm{RE})$ foi obtida pela diferença entre a $R E$ determinada na segunda época $\left(E_{2}\right.$ - setembro de 2008) e a RE da primeira ( $E_{1}$ - outubro de 2007). Da mesma forma, determinou-se a variação temporal do estoque de $\operatorname{COT}(\Delta \mathrm{COT})$, que corresponde à taxa de sequestro no solo.

Análise estatística

Os ensaios foram desenvolvidos em delineamento inteiramente casualizado com doze repetições. Os resultados obtidos foram submetidos ao teste de Tukey a 5\% de significância, com o software SASM Agri (CANTERI et al., 2001). Para a obtenção das curvas de respostas, foi utilizado o procedimento da análise de regressão, utilizando-se o programa JMP $\mathrm{IN}^{\circledR}$ Version 3.2.1 (SALL et al., 2005), com o teste F a 5\% de significância.

\section{RESULTADOS E DISCUSSÃO}

Conteúdo de carbono e relação de estratificação No solo com classe textural franco-argiloarenosa (LVTM), o conteúdo médio do COT na camada $0-5 \mathrm{~cm}$ apresentou um incremento de $26,61 \%$ em relação à camada $5-20 \mathrm{~cm}$. Nesse mesmo solo, o conteúdo médio do COP na camada $0-5 \mathrm{~cm}$ apresentou um incremento de 52,89\% em relação à camada $5-20 \mathrm{~cm}$ (Tabela 2). No solo com classe textural franco-argilosa (LVTA), os conteúdos médios de COT e COP não apresentaram diferenças significativas entre as camadas.

$\mathrm{Na}$ classe de agregados com diâmetro entre 19-8mm, observou-se, na camada de 0-5 cm do LVTM, um incremento de $9,60 \%$ no teor de $\mathrm{C}$ da primeira para segunda época de coleta, enquanto, na camada $5-20 \mathrm{~cm}$, esse incremento foi de $16,38 \%$ (Tabela 2). Constatouse, na classe de agregados entre $0,25-0,5 \mathrm{~mm}$, menores valores de $\mathrm{C}$ nos dois compartimentos analisados (COT e COP) em ambas as épocas e camadas do LVTA, porém, no LVTM, isso ocorreu apenas para o compartimento de COT (Tabela 2). Esses resultados são semelhantes aos encontrados por CANALLI (2009) na mesma região de estudo, na qual o autor observou aumento do COT nos macroagregados (diâmetro entre 19-8mm). Em outras regiões do país, esse efeito também foi constatado. ASSIS et al. (2006), em Minas Gerais, e NICOLOSO (2009), no Rio Grande do Sul, observaram teores mais elevados de COT nos macroagregados maiores do que $4 \mathrm{~mm}$.

No presente estudo, fica evidente o incremento de $\mathrm{C}$ nos macroagregados no solo manejado sob SPD por longo prazo, principalmente na classe de 8-19mm de diâmetro (Tabela 2). BAYER et al. (2011) afirmaram que o aumento da macroaggregação melhorou a estabilização e, consequentemente, a fixação de $\mathrm{C}$ dentro dos agregados. Isso ocorre porque, no SPD, não ocorre o constante fraturamento dos macroagragados, provocado pelo preparo mecânico do solo, ou este fica restrito às linhas de semeadura. Em consequência, a entrada de $\mathrm{O}$ através das trocas gasosas com o ar atmosférico é señivivelmente reduzida, criando um ambiente menos oxidativo no interior dos agregados. Assim, a exposição da MOS ao ataque microbiano é minimizada, permitindo que os agentes de agregação atuem como ligantes na formação de macroagregados (BAYER et al., 2011).

Considerando a média de todas as classes de agregados estudadas neste experimento, observase uma variação da RE do COT de 1,07 a 1,60 e do COP 
Tabela 2 - Conteúdo de carbono e relação de estratificação nos compartimentos COT e COP das classes de agregados nos dois Latossolos estudados.

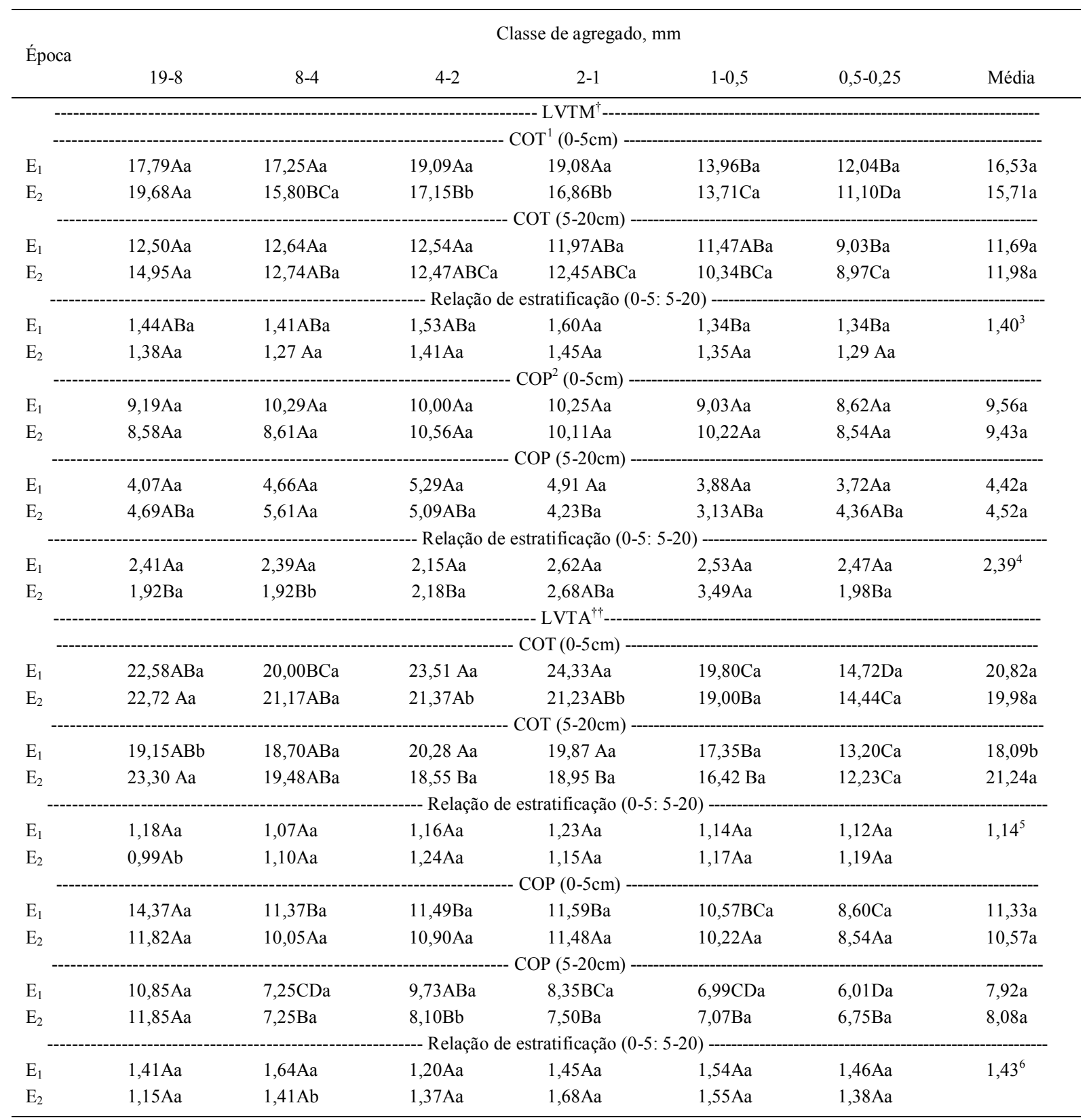

${ }^{\dagger}$ Latossolo Vermelho Distrófico típico, com classe textural franco argilo arenosa; ${ }^{\dagger}$ Latossolo Vermelho Distrófico típico, com classe textural franco argilosa. ${ }^{1} \mathrm{COT}$-carbono orgânico total; ${ }^{2} \mathrm{COP}$-carbono orgânico particulado. Médias seguidas por letras iguais, minúsculas nas colunas e maiúsculas nas linhas, não diferem entre si pelo teste de Tukey, a 5\% de significância. Comparação entre épocas na coluna e comparação entre classes de agregados na linha. ${ }^{3} \mathrm{Média}=\left(\mathrm{RE}\right.$ do COT da $\mathrm{E}_{1}+\mathrm{RE}$ do COT da $\mathrm{E}_{2}$ da LVTM $) / 2 ;{ }^{4} \mathrm{Média}=\left(\mathrm{RE}\right.$ do COP da $\mathrm{E}_{1}+\mathrm{RE}$ do COP da $\mathrm{E}_{2}$ da LVTM $) / 2 ;{ }^{5} \mathrm{Média}=\left(\mathrm{RE}\right.$ do COT $\mathrm{E}_{1}+\mathrm{RE}$ do COT $\mathrm{E}_{2}$ da LVTA $) / 2 ;{ }^{6} \mathrm{Média}=\left(\mathrm{RE}\right.$ do COP $\mathrm{E}_{1}+\mathrm{RE}$ do COP $\mathrm{E}_{2}$ da LVTA $) / 2$.

de 1,15 a 3,49. Como o princípio da RE é comparar uma camada superficial com elevada influência das práticas de manejo antrópicas com outra camada com baixo impacto dessas práticas, as variações na RE dentro dos compartimentos (COT e COP) indica o grau de organização e funcionamento dos diferentes ecossistemas. Na classe dos macroagregados com diâmetro de 8-19mm, observa-se uma média geral da RE do COT de 1,22 $( \pm 0,23)$, observações estas rafiticadas por CANALLI (2009), que, em SPD de longa 
duração, na mesma região do estudo, também encontrou a média de 1,23 de RE.

A RE média de COT no LVTM foi de 1,40 $( \pm 0,31)$, enquanto que no LVTA foi de $1,14( \pm 0,24)$, enquanto a RE média de COP no LVTM foi de 2,39 $( \pm 1,57)$ e no LVTA foi de $1,43( \pm 0,53)$ (Tabela 2$)$. Esse comportamento indica que, no LVTM, a taxa de conversão do $\mathrm{C}$ dos resíduos culturais foi superior à do LVTA. Isso indica que a ação dos agentes temporários (raízes e hifas de fungos), assim como a quantidade de carbono liberado por essas raízes no ambiente interno dos agregados, garantiu maior intensidade de formação destes macroagregados e, em consequência, maior proteção do carbono (SÁ et al., 2010; BAYER et al., 2011; VEZZANI \& MIELNICZUK (2011). A ação desses agentes temporários, em especial de fungos micorrízicos arbusculares, em áreas manejadas com SPD de longa duração, representa um importante fator de incremento da recuperação de $\mathrm{C}$ adicionado nos macroagregados (WHITE \& RICE, 2009).

Relação de estratificação e a taxa de sequestro de C

A correlação entre o delta da relação estratificação e a taxa de sequestro de $\mathrm{C}$ na camada de $0-5 \mathrm{~cm}$ foi significativa em ambos os solos estudados (Figura 1). Resultados semelhantes foram obtidos por

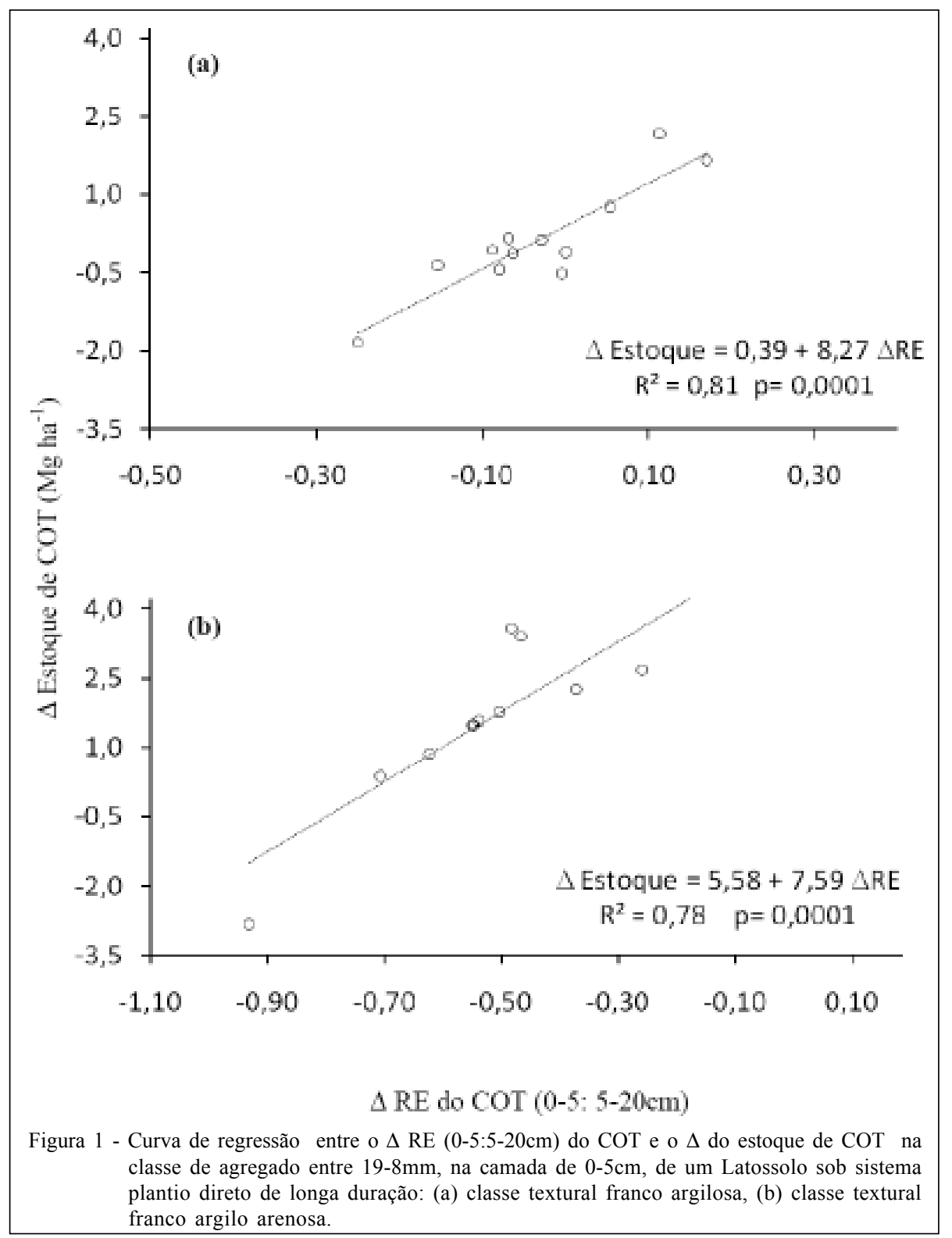

Ciência Rural, v.42, n.4, abr, 2012. 
CANALLI (2009), que também constatou esse efeito nos macroagregados com diâmetro de $19-8 \mathrm{~mm}$, demonstrando que a RE acompanhou o aumento do estoque de COT no solo, confirmando, assim, a hipótese sobre o uso deste parâmetro como indicador do sequestro de $\mathrm{C}$ no solo manejado sob SPD. O SPD contribuiu diretamente para a adição continua de carbono pelos resíduos orgânicos e sua decomposição, enriquecendo a camada superficial do perfil do solo. Segundo SÁ \& LAL (2009) e FRANZUEBBERS (2010), a elevada RE de C reflete diretamente ao solo uma alta qualidade superficial, que aumenta a infiltração da água no perfil e a estabilidade de agregados.

Segundo SÁ et al. (2010), a maior porcentagem de COT e COP nas classes de macroagregados maiores que $8 \mathrm{~mm}$ tem forte influência da atividade de raízes e de fungos saprofíticos, por causa das condições favoráveis que a cobertura do solo promove. Os autores afirmam que esse aumento da porcentagem dos macroagregados indica melhor qualidade da estrutura do solo, pela formação de mais planos de fraqueza por onde as raízes exploram o espaço poroso.

No compartimento de COT, a equação de regressão entre o $\Delta$ RE e o $\Delta$ do conteúdo de COT no LVTM foi significativa nas classes de agregados com diâmetro de 19-8, 8-4, 4-2, 1-0,5 e 0,5-0,25mm. Por outro lado, no LVTA, essa relação somente foi significativa para as classes de agregados com diâmetro de 19-8 e 0,5-0,25mm. No compartimento de COP, o LVTM apresentou relação significativa nos agregados das classes de 19-8, 4-2, 1-0,5 e 0,5-0,25mm de diâmetro. No LVTA, essa relação não foi significativa somente para os agregados da classe de $2-1 \mathrm{~mm}$ de diâmetro (Tabela $3)$. Esses resultados evidenciam que o conteúdo de COT e COP está intimamente relacionado com a estratificação do $\mathrm{C}$ dentro das classes de agregados desses compartimentos.

Tabela 3 - Equações de regressão entre a variação do $\operatorname{COT}\left(\Delta \mathrm{COT}^{\S}\right)$ e variação do $\operatorname{COP}\left(\Delta \mathrm{COP}^{\S}\right) \operatorname{com}^{\mathrm{a}}$ delta da $\mathrm{RE}^{\S \S}$.

\begin{tabular}{|c|c|c|c|c|c|}
\hline Comp.(tipo) & Classe textural & Classe de agregado (mm) & Equação & $\mathrm{R}^{2}$ & Nível de significância (p) \\
\hline \multirow[t]{12}{*}{$\mathrm{COT}$} & \multirow[t]{6}{*}{$\operatorname{LVTM}^{\dagger}$} & $19-8$ & $\Delta \mathrm{COT}=5,80+6,71 \Delta \mathrm{RE}$ & 0,43 & 0,02 \\
\hline & & $8-4$ & $\Delta \mathrm{COT}=-0,76+4,98 \Delta \mathrm{RE}$ & 0,31 & 0,06 \\
\hline & & $4-2$ & $\Delta \mathrm{COT}=-1,16+6,10 \Delta \mathrm{RE}$ & 0,36 & 0,04 \\
\hline & & $2-1$ & $\Delta \mathrm{COT}=-193+1,89 \Delta \mathrm{RE}$ & 0,12 & 0,26 \\
\hline & & $1-0,5$ & $\Delta \mathrm{COT}=-0,28+3,96 \Delta \mathrm{RE}$ & 0,25 & 0,09 \\
\hline & & $0,5-0,25$ & $\Delta \mathrm{COT}=-0,68+5,12 \Delta \mathrm{RE}$ & 0,62 & 0,002 \\
\hline & \multirow[t]{6}{*}{ LVTA $^{\dagger \dagger}$} & $19-8$ & $\Delta \mathrm{COT}=1,49+7,31 \Delta \mathrm{RE}$ & 0,40 & 0,02 \\
\hline & & $8-4$ & $\Delta \mathrm{COT}=1,08+4,14 \Delta \mathrm{RE}$ & 0,16 & 0,18 \\
\hline & & $4-2$ & $\Delta \mathrm{COT}=-2,26+1,56 \Delta \mathrm{RE}$ & 0,09 & 0,35 \\
\hline & & $2-1$ & $\Delta \mathrm{COT}=-2,76+4,70 \Delta \mathrm{RE}$ & 0,14 & 0,21 \\
\hline & & $1-0,5$ & $\Delta \mathrm{COT}=-0,89+2,53 \Delta \mathrm{RE}$ & 0,03 & 0,55 \\
\hline & & $0,5-0,25$ & $\Delta \mathrm{COT}=-0,55+3,60 \Delta \mathrm{RE}$ & 0,40 & 0,02 \\
\hline \multirow[t]{12}{*}{$\mathrm{COP}$} & \multirow[t]{6}{*}{ LVTM } & $19-8$ & $\Delta \mathrm{COP}=0,18+1,60 \Delta \mathrm{RE}$ & 0,28 & 0,07 \\
\hline & & $8-4$ & $\Delta \mathrm{COP}=-0,83+1,78 \Delta \mathrm{RE}$ & 0,18 & 0,17 \\
\hline & & $4-2$ & $\Delta \mathrm{COP}=0,49+2,46 \Delta \mathrm{RE}$ & 0,64 & 0,001 \\
\hline & & $2-1$ & $\Delta \mathrm{COP}=-0,20+1,09 \Delta \mathrm{RE}$ & 0,17 & 0,18 \\
\hline & & $1-0,5$ & $\Delta \mathrm{COP}=-0,36+1,59 \Delta \mathrm{RE}$ & 0,47 & 0,01 \\
\hline & & $0,5-0,25$ & $\Delta \mathrm{COP}=1,26+2,74 \Delta \mathrm{RE}$ & 0,54 & 0,006 \\
\hline & \multirow[t]{6}{*}{ LVTA } & $19-8$ & $\Delta \mathrm{COP}=-1,70+3,23 \Delta \mathrm{RE}$ & 0,41 & 0,01 \\
\hline & & $8-4$ & $\Delta \mathrm{COP}=-0,77+2,37 \Delta \mathrm{RE}$ & 0,25 & 0,09 \\
\hline & & $4-2$ & $\Delta \mathrm{COP}=-1,46+5,20 \Delta \mathrm{RE}$ & 0,49 & 0,01 \\
\hline & & $2-1$ & $\Delta \mathrm{COP}=-0,29+0,79 \Delta \mathrm{RE}$ & 0,04 & 0,51 \\
\hline & & $1-0,5$ & $\Delta \mathrm{COP}=-0,38+2,92 \Delta \mathrm{RE}$ & 0,44 & 0,01 \\
\hline & & $0,5-0,25$ & $\Delta \mathrm{COP}=-0,15+2,77 \Delta \mathrm{RE}$ & 0,56 & 0,005 \\
\hline
\end{tabular}

${ }^{\S}$ Delta COT $(\Delta \mathrm{COT})$ e delta COP $(\Delta \mathrm{COP})$ representa o $\mathrm{C}$ acumulado calculado pela diferença do estoque: estoque de $\mathrm{C}$ na $\mathrm{E}_{2}-$ estoque de $\mathrm{C}$ na $\mathrm{E}_{1} ;{ }^{\S \S}$ delta da RE $(0-5: 5-20 \mathrm{~cm})$ representa a variação da $\mathrm{RE}$ entre a $\mathrm{E}_{2}-\mathrm{E}_{1}$. ${ }^{\dagger}$ Latossolo Vermelho Distrófico típico, com classe textural franco argilo arenosa $;{ }^{\dagger}$ Latossolo Vermelho Distrófico típico, com classe textural franco argilosa. 
No compartimento particulado, essa relação foi mais acentuada, principalmente no LVTA, pois, conforme BAYER et al. (2011), a estabilização da MOP depende fortemente da textura do solo, ou seja, com aumento no teor de argila, ocorre um incremento no teor de $\mathrm{C}$ no interior dos agregados, favorecendo, dessa forma, o acúmulo de carbono.

\section{CONCLUSÃO}

A correlação linear significativa entre o delta da relação de estratificação do carbono orgânico total e particulado com o delta do estoque de carbono indicou aumento no sequestro de carbono nos macroagregados nos dois solos estudados. Os resultados obtidos permitem confirmar a hipótese da relação de estratificação ser um indicador sensível para a avaliação da taxa de sequestro de carbono orgânico total e particulado em macroagregados do solo manejado sob sistema plantio direto.

\section{REFERÊNCIAS}

ASSIS, C.P. et al. Carbon and nitrogen in aggregates of an Oxisol submitted to different use and management systems. Pesquisa agropecuária Brasileira, v.41, n.10, p.1541-1550, 2006 Disponível em: $<$ http://www.scielo.br/scielo.php?pid=s0100$204 \times 2006001000012 \&$ script $=$ sci arttext $>$. Acesso em: 14 abr. 2011. doi: 10.1590/S0100-204X2006001000012.

BARRETO, R.C. et al. The impact of soil management on aggregation, carbon stabilization and carbon loss as $\mathrm{CO}$ in the surface layer of a Rhodic Ferralsol in Southern Brazil. Agriculture, Ecosystems \& Environment, v.132, p.243251, 2009. Disponível em: <http://www.sciencedirect.com/ science/article/pii/S0167880909001091>. Acesso em: 14 abr. 2011. doi: 10.1016/j.agee.2009.04.008.

BAYER, C. et al. Estabilização do carbono no solo e mitigação das emissões de gases de efeito estufa na agricultura conservacionista. In: KLAUBERG FILHO, O.; MAFRA, A.L.; GATIBONI, L.C. (Eds.). Tópicos em ciência do solo. Viçosa: Sociedade Brasileira de Ciência do Solo. 2011. V.VII, cap.2, p.55-118.

CASTRO FILHO, C. et al. Estabilidade dos agregados e sua relação com o teor de carbono orgânico num Latossolo Roxo distrófico, em função de sistemas de plantio, rotações de culturas e métodos de preparo das amostras. Revista Brasileira de Ciência do Solo, v.22, n.3, p.527-538, 1998. Disponível em: <http://sbcs.solos.ufv.br/solos/revistas/v22n3a19.pdf $>$. Acesso em: 14 abr. 2011.

CANALLI, L.B.S. Decomposição de resíduos culturais e sua contribuição nos macroagregados e na fração lábil da matéria orgânica do solo no sistema plantio direto.
2009. 109f. Tese (Doutorado em Agronomia) - Programa de Pós-graduação em Produção Vegetal, Universidade Federal do Paraná, PR.

CANTERI, M.G. et al. SASM-Agri - Sistema para análise e separação de médias em experimentos agrícolas pelos métodos Scott-Knott, Tukey e Duncan. Revista Brasileira de Agrocomputação, v.1, n2, p.18-24, 2001. Disponível em: $<$ http://www.agrocomputacao.deinfo.uepg.br/dezembro_2001/ Arquivos/RBAC_Artigo_03.pdf>. Acesso em: 05 dez. 2011.

EMPRESA BRASILEIRA DE PESQUISA AGROPECUÁRIA EMBRAPA. Manual de métodos de análise do solo. 2. ed. Rio de Janeiro, RJ, 1997. p.212.

EMPRESA BRASILEIRA DE PESQUISA AGROPECUÁRIA EMBRAPA - Sistema Brasileiro de Classificação de Solos. 2.ed. Rio de Janeiro. Embrapa, 2006. 306p.

FRANZLUEBBERS, A.J. Soil organic matter stratification ratio as an indicator of soil quality. Soil and Tillage Research, v.66, p.95-106, 2002. Disponível em: <http://www.sciencedirect.com/ science/article/pii/S0167198702000181>. Acesso em: 14 abr. 2011. doi: 10.1016/S0167-1987(02)00018-1.

FRANZLUEBBERS, A.J. Achieving soil organic carbon sequestration with conservation agricultural systems in the southeastern USA. Soil Science Society of America Journal, v.74, n.2, p.347-357, 2010. Disponível em: <http:/ /ddr.nal.usda.gov/bitstream/10113/41306/1/ IND44334724.pdf>. Acesso em: 14 abr. 2011. doi: 10.2136/ sssaj2009.0079.

FELLER, C. La matière organique dans le sols tropicaux à argiles 1:1. Recherche de compartiments organiques fontionnels. Une approche granulometrique. 1994. 393f. Thèse (Doctorate) - Universitè Strasbourg.

FERREIRA, A.O. et al. Variação na resistência tênsil de agregados em função do conteúdo de carbono em dois solos na região dos Campos Gerais. Revista Brasileira de Ciência do Solo, v.35, n.2, p.437-446, 2011. Disponível em: <http:/ /www.scielo.br/pdf/rbcs/v35n2/v35n2a13.pdf $>$. Acesso em: 14 abr. 2011. doi: 10.1590/S0100-06832011000200013.

INSTITUTO AGRONOMICO DO PARANA - IAPAR. Dados climatológicos de Ponta Grossa - 2008. Ponta Grossa, 2008. 22p.

NICOLOSO, R.S. Estoques e mecanismos de estabilização da matéria orgânica do solo em agroecossistemas de clima temperado e subtropical. 2009. 109f. Tese (Doutorado em Engenharia Agrícola) - Programa de Pósgraduação em Engenharia Agrícola, Universidade Federal de Santa Maria, RS

PAVAN, M.A. et al. Manual de análise química do solo e controle de qualidade. Curitiba: Instituto Agronômico do Paraná, 1992. 38p. (Circular, 76).

SÁ, J.C.M.; LAL, R. Stratification ratio of soil organic matter pools as an indicator of carbon sequestration in a tillage chronosequence on a Brazilian Oxisol. Soil and Tillage Research, v.103, p.46- 
56, 2009. Disponível em: <http://www.sciencedirect.com/science/ article/pii/S0167198708001736>. Acesso em: 14 abr. 2011. doi: 10.1016/j.still.2008.09.003.

SÁ, J.C.M. et al. Gestão da matéria orgânica e da fertilidade do solo visando sistemas sustentáveis de produção. In: PROCHNOW, L.I. et al. (Org.). Boas práticas para uso eficiente de fertilizantes. Piracicaba, SP: International Plant Nutrition Institute - Brasil (IPNI), 2010. V.1, p.383-420.

SALL, J. et al. JMP start statistics: a guide to statistics and data analysis using JMP and JMP IN software. 3.ed. Cary: Duxbury, 2005. 580p.
VEZZANI, F.M.; MIELNICZUK, J. Agregação e estoque de carbono em argissolo submetido a diferentes práticas de manejo agrícola. Revista Brasileira de Ciência do Solo, v.35, n.1, p.213-223, 2011. Disponível em: <http://www.scielo.br/pdf/ rbcs/v35n1/a20v35n1.pdf $>$. Acesso em: 14 abr. 2011. doi: 10.1590/S0100-06832011000100020.

WHITE, P.; RICE, C.W. Tillage effects on microbial and carbon dynamics during plant residue decomposition. Soil Biology \& Biochemistry, v.73, n.1, p.138-145, 2009. Disponível em: $<$ https://www.soils.org/publications/sssaj/articles/73/1/138>. Acesso em: 14 abr. 2011. doi: 10.2136/sssaj2007.0384. 